\section{Macular thickness reduction in eyes with unilateral optic atrophy detected with optical coherence tomography}

S Kusuhara, M Nakamura, A Nagai-Kusuhara, Y Nakanishi, A Kanamori and A Negi
Department of Organ Therapeutics, Division of Ophthalmology, Kobe University Graduate School of Medicine, Chuo-Ku, Kobe, Japan

Correspondence:

S Kusuhara, Department of Organ Therapeutics,

Division of Ophthalmology, Kobe University Graduate School of Medicine, 7-5-2 Kusunoki-Cho, Chuo-Ku, Kobe, Japan

Tel: + 81783826048 Fax: + 81783826059

E-mail: kusu@

med.kobe-u.ac.jp

Received: 28 February 2005 Accepted: 10 June 2005 Published online: 15 July 2005

We have no proprietary interest or research funding in this study

\section{Abstract}

Aims To assess the changes in macular and peripapillary retinal nerve fibre layer (RNFL) thickness in eyes with unilateral optic atrophy and to evaluate the relationship between retinal thickness and visual function. Methods Enrolled were 22 patients with unilateral optic atrophy. Macular thickness at the divided nine areas and peripapillary RNFL thickness in quadrantic sections were measured by optical coherence tomography. Thickness values in the affected eyes were compared with those in the contralateral unaffected eyes. The correlation of foveal thickness with best-corrected visual acuity (BCVA) was evaluated. The correlation between retinal thickness and the remaining visual field area circumscribed with I-4-e isopter in superior and inferior hemifield was assessed.

Results Macular thinning was observed in all areas $(P<0.001$ in each area) other than the fovea $(P=0.068)$. Peripapillary RNFL thickness decreased in all quadrantic sections $(P<0.001$ in each section). The affected to unaffected eye ratio of retinal thickness was more than 0.6 in each area. BCVA did not correlate with foveal thickness (correlation coefficient $=0.094, P=0.668)$. Although not statistically significant $(P=\mathbf{0 . 2 8 1}$, superior hemifield; $P=0.053$, inferior hemifield), there was a tendency that eyes with severe visual field loss show more marked retinal thinning. Conclusions Macular thinning with the preserved foveal thickness is a hallmark of eyes with optic atrophy. Together with no correlation between foveal thickness and BCVA, this finding would help in differential diagnosis of macular and optic nerve diseases.

Eye (2006) 20, 882-887. doi:10.1038/sj.eye.6702013; published online 15 July 2005

Keywords: optic atrophy; retinal thickness; optical coherence tomography; macula

\section{Introduction}

Optic atrophy is a common end point finding of pathologies affecting the anterior visual pathway up to the lateral geniculate body and essentially reflects loss of retinal ganglion cell axons. ${ }^{1}$ Axonal damage retrogradely degenerates ganglion cell body. Since the ganglion cells and retinal nerve fibre layer (RNFL) contribute to retinal thickness in the macula, ${ }^{2}$ macular thickness is expected to be reduced to some degree in eyes with optic atrophy.

Optical coherence tomography (OCT) is an imaging device and confers both objective and quantitative information on macular and RNFL thickness with 10-15 $\mu$ m vertical resolution.,4 OCT has been mainly used for evaluating various types of retinal diseases, glaucoma, and optic nerve diseases. ${ }^{5-14}$ In eyes with macular disease such as occult macular dystrophy, and various types of macular oedema, foveal thickness have shown a correlation with visual acuity, and alterations of macular thickness have been adopted in assessing progress of disease. ${ }^{15-18}$ On the other hand, some studies remarked and demonstrated the decrease in peripapillary RNFL thickness in eyes with glaucoma, traumatic optic neuropathy, and compression optic neuropathy using OCT. ${ }^{9-14,19,20}$ 
Although it has been reported that peripapillary RNFL thickness represents the severity of visual field loss and would be of clinical use, ${ }^{14,21}$ little attention have been paid to the relationship between visual function and macular thickness in eyes with optic nerve disease. The purpose of the current study was to clarify the detailed characteristics of retinal damages in the macular regions due to optic nerve diseases. For this purpose, we evaluated the reduction of OCT-measured macular and peripapillary RNFL thickness in eyes with unilateral optic atrophy and assessed the relationship between retinal thickness and visual acuity or visual field.

\section{Materials and methods}

After obtaining written informed consent, 22 patients with unilateral optic atrophy were enrolled between June 2003 and December 2004. All participants underwent visual acuity test, Goldmann kinetic perimetry, stereoscopic slit-lamp examination for the macula and optic disc evaluations, and OCT. Patients were excluded from this study if they had severe opacification of optic media, previous and/or coexisting macular disease or glaucoma, or systemic diseases or drug intake that may affect the retinal or RNFL thickness.

This study was performed in accordance with the Tenets of Declaration of Helsinki and was approved by the ethical committee of Kobe University Graduate School of Medicine.

Best-corrected visual acuity (BCVA) was measured by standard Snellen visual acuity charts and converted to the $\log$ MAR scale in statistical analyses. In this conversion, visual acuities of hand motion and light perception were assigned to 20/2000 and 20/20000 of the Snellen fraction, respectively.

Macular thickness and RNFL thickness around the optic disc were measured by OCT (OCT1,

Zeiss-Humphrey, Dublin, California), as reported previously. ${ }^{13,22}$ In brief, retinal thickness was calculated using OCT retinal mapping software (version 6.1a), which measured thickness of nine divided macular regions (A0-A8) after six scans with $6 \mathrm{~mm}$ long under pupillary dilation and expressed as a colour-coded map. RNFL thickness was measured by 3.4-mm-diameter circle scans centred on the optic disc. RNFL thickness was obtained and averaged from the values of three scans in each quadrantic section. Internal fixation was essentially selected due to better reproducibility, although external fixation was chosen in six subjects with severely impaired visual acuity, equal to or less than $20 / 200$, in the affected eye. Defined area location in the OCT measurement is presented in Figure 1.

Analyses of the obtained data were carried out as follows. First, to verify whether ganglion cell loss in

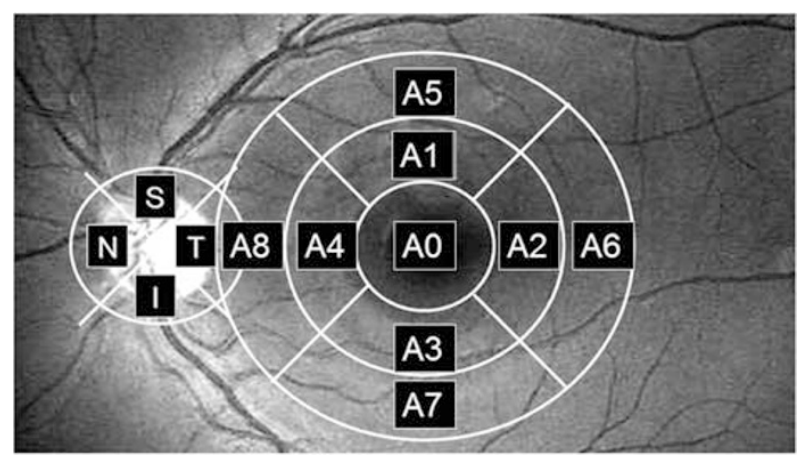

Figure 1 Defined area location in the analyses. The macula was divided into three concentric areas. A0 is the foveal region measuring $1.00 \mathrm{~mm}$ in diameter. The inner and outer rings are each divided into four quadrants (A1-A4 and A5-A8); the rings have diameters of 2.22 and $3.45 \mathrm{~mm}$, respectively. A $3.4-\mathrm{mm}$ diameter circle centred on the optic disc is divided into four quadrantic sections ( $\mathrm{S}$, superior; $\mathrm{T}$, temporal; $\mathrm{I}$, inferior; $\mathrm{N}$, nasal).

optic atrophy affects macular thickness, comparison of macular thickness as well as peripapillary RNFL thickness between affected and unaffected eyes was performed. Next, to quantify the degree of decrease in macular thickness of eyes with optic atrophy, the ratio of retinal thickness in affected eyes relative to that in unaffected eyes was calculated in each area. Third, whether foveal thickness is responsible to visual acuity in eyes with optic atrophy was determined by assessing the relationship between logMAR BCVA and foveal thickness. Finally, we assessed the relationship between the degree of visual field loss and retinal thickness at the corresponding area. The degree of field loss was scored in superior and inferior hemifields as the remaining area circumscribed with I-4-e isopter within $15^{\circ}$, approximately comparable to the OCTmeasured circle area with a diameter of $6 \mathrm{~mm}$, that is, $0-25 \%$ ( $n=6$, superior hemifield; $n=6$, inferior hemifield), $25-50 \%$ ( $n=1$, superior hemifield; $n=2$, inferior hemifield), $50-75 \%$ ( $n=1$, superior hemifield; $n=2$, inferior hemifield), and 75-100\% ( $n=14$, superior hemifield; $n=12$, inferior hemifield). Retinal thickness at the area corresponding to each hemifield was obtained.

Statistical analyses were performed using Stat View version 5.0 software (SAS Institute, Cary, NC, USA). Wilcoxon's signed-rank test was used to compare averages of parameters between affected and unaffected eyes. Association between foveal thickness and BCVA was evaluated by Spearman's analysis. Comparison of retinal thickness among eyes with various residual visual fields was assessed by Kruskal-Wallis test. $P$-value less than 0.05 was considered to be statistically significant. 


\section{Results}

Of the 22 patients, $12(54.5 \%)$ were female and 10 (45.5\%) were male. The mean age and standard deviation (SD) was 56.3 (4.33) years, ranging 11-79, and the mean (SD) duration after the onset of optic neuropathy was 5.09 (4.26) years, ranging 1-14. Aetiology of optic neuropathy was unknown in seven cases $(31.8 \%)$, ischaemia in six $(27.3 \%)$, trauma in three $(13.6 \%)$, retrobulbar optic neuritis in three (13.6\%), and others in three (13.6\%).

BCVA ranged from light perception to $20 / 20$, and the mean (SD) logMAR-converted BCVA was 0.701 (1.177). The patterns of visual field defect were central or cecocentral scotoma in five eyes $(22.7 \%)$, peripheral island in four eyes (18.2\%), altitudinal hemianopia in four eyes (18.2\%), quadrantic hemianopia in four eyes $(18.2 \%)$, normal visual field in four eyes (18.2\%), and temporal hemianopia in one eye $(4.5 \%)$. Clinical characteristics of the participants are described in Table 1. In all subjects, unaffected fellow eyes had BCVA of 20/20 (0 logMAR unit) and unimpaired visual field.

Statistically significant decrease in RNFL thickness of the affected eyes was observed in all quadrantic sections $(P<0.001)$. Decrease in retinal thickness of the affected eye was significant in all areas except for A0, foveal area $(P<0.001$; Table 2, Figures 2 and 3). Retinal thickness in the affected eyes was at least $60 \%$ of the value of that in the unaffected fellow eyes (Table 3), whereas RNFL thickness reduction in some cases exceeded $90 \%$ of contralateral eyes. There was no significant correlation between logMAR BCVA and foveal thickness (correlation

Table 2 Mean values (SD) of macular or RNFL thickness $(\mu \mathrm{m})$ in each segment for the 22 eyes with optic atrophy and 22 unaffected fellow eyes

\begin{tabular}{lcrr}
\hline & $\begin{array}{c}\text { Optic atrophy } \\
(\mathrm{n}=22)\end{array}$ & $\begin{array}{c}\text { Fellow eye } \\
(\mathrm{n}=22)\end{array}$ & \multicolumn{1}{c}{ P-value $^{*}$} \\
\hline RNFL quadrants & & & \\
$\quad$ Superior & $87.2(42.9)$ & $138.0(28.8)$ & 0.0001 \\
Temporal & $53.1(35.0)$ & $93.1(25.1)$ & $<0.0001$ \\
Inferior & $87.2(34.5)$ & $140.6(20.9)$ & $<0.0001$ \\
Nasal & $49.3(32.1)$ & $84.3(36.0)$ & $<0.0001$ \\
& & & \\
Macular area & & & \\
A0 & $192.7(25.9)$ & $205.9(28.7)$ & 0.0680 \\
A1 & $225.4(35.0)$ & $260.5(27.5)$ & 0.0001 \\
A2 & $217.1(25.8)$ & $244.2(28.7)$ & 0.0002 \\
A3 & $224.4(29.7)$ & $261.5(21.3)$ & $<0.0001$ \\
A4 & $227.5(39.0)$ & $264.1(26.7)$ & 0.0005 \\
A5 & $218.1(35.6)$ & $250.4(24.2)$ & $<0.0001$ \\
A6 & $209.4(37.2)$ & $228.1(40.7)$ & 0.0078 \\
A7 & $209.6(29.7)$ & $240.3(26.3)$ & 0.0001 \\
A8 & $223.5(34.7)$ & $260.0(21.7)$ & $<0.0001$ \\
\hline
\end{tabular}

*Wilcoxon's signed-rank test.

Table 1 Patient demographics

\begin{tabular}{|c|c|c|c|c|c|c|c|c|}
\hline Case no. & Age (years) & Sex & Eye & Diagnosis & $\begin{array}{c}\text { Duration } \\
\text { (years) }\end{array}$ & Visual acuity & Visual field & Systemic disease \\
\hline 1 & 72 & M & OD & ION & 12 & $20 / 30$ & Altitudinal hemianopia & Hyperlipidaemia \\
\hline 2 & 65 & $\mathrm{~F}$ & OS & $\mathrm{RON}$ & 12 & LP & Peripheral island & \\
\hline 3 & 68 & M & OD & ION & 2 & $20 / 25$ & Quadrantic hemianopia & \\
\hline 4 & 66 & $\mathrm{~F}$ & OD & TON & 14 & $20 / 30$ & Altitudinal hemianopia & \\
\hline 5 & 68 & $\mathrm{M}$ & OS & $\mathrm{CON}$ & 1 & $20 / 20$ & Normal & Brain infarction, DM, HT \\
\hline 6 & 57 & $\mathrm{M}$ & OS & Papillitis & 1 & $20 / 20$ & Normal & DM \\
\hline 7 & 42 & $\mathrm{~F}$ & OD & ION & 1 & $20 / 100$ & Altitudinal hemianopia & HT \\
\hline 8 & 40 & $\mathrm{M}$ & OS & $\mathrm{OA}$ & 5 & HM & Peripheral island & \\
\hline 9 & 67 & M & OS & ION & 5 & $20 / 20$ & Cecocentral scotoma & Tuberculosis, TAO \\
\hline 10 & 74 & $\mathrm{~F}$ & OD & RON & 4 & $20 / 20$ & Normal & Hyperlipidaemia \\
\hline 11 & 11 & M & OD & $\mathrm{OA}$ & 4 & $20 / 20$ & Relative central scotoma & \\
\hline 12 & 42 & M & OD & $\mathrm{OA}$ & 5 & $20 / 20$ & Normal & \\
\hline 13 & 79 & $\mathrm{~F}$ & OS & $\mathrm{OA}$ & 5 & $20 / 500$ & Central scotoma & HT \\
\hline 14 & 21 & $\mathrm{~F}$ & OS & Meningioma & 5 & $20 / 20$ & Quadrantic hemianopia & \\
\hline 15 & 70 & $\mathrm{~F}$ & OD & $\mathrm{OA}$ & 13 & $\mathrm{HM}$ & Peripheral island & \\
\hline 16 & 66 & $\mathrm{~F}$ & OS & RON & 3 & $20 / 25$ & Quadrantic hemianopia & Aplastic anaemia \\
\hline 17 & 19 & $\mathrm{~F}$ & OD & $\mathrm{OA}$ & 1 & $20 / 20$ & Cecocentral scotoma & \\
\hline 18 & 66 & $\mathrm{~F}$ & OD & TON & 5 & $20 / 20$ & Quadrantic hemianopia & HT \\
\hline 19 & 52 & $\mathrm{~F}$ & OS & $\mathrm{OA}$ & 1 & $20 / 500$ & Temporal hemianopia & \\
\hline 20 & 68 & $\mathrm{~F}$ & OD & ION & 1 & $20 / 200$ & Peripheral island & \\
\hline 21 & 51 & M & OS & $\mathrm{ION}$ & 1 & $20 / 20$ & Cecocentral scotoma & DM, HT \\
\hline 22 & 71 & M & OD & TON & 7 & $20 / 30$ & Altitudinal hemianopia & \\
\hline
\end{tabular}

$\mathrm{F}=$ female; $\mathrm{M}=$ male; $\mathrm{OD}=$ right eye; $\mathrm{OS}=$ left eye; $\mathrm{ION}=$ ischaemic optic neuropathy; $\mathrm{RON}=$ retrobulbar optic neuritis; TON = traumatic optic neuropathy; $\mathrm{CON}=$ compressive optic neuropathy; $\mathrm{OA}=$ optic atrophy of unknown origin; meningioma = parasellar meningioma; $\mathrm{LP}=$ light perception; $\mathrm{HM}=$ hand motion; $\mathrm{HT}=$ hypertension; $\mathrm{DM}=$ diabetes mellitus; $\mathrm{TAO}=$ thromboangiitis obliterans. Durations indicate the period from the onset of disease to OCT examination. 


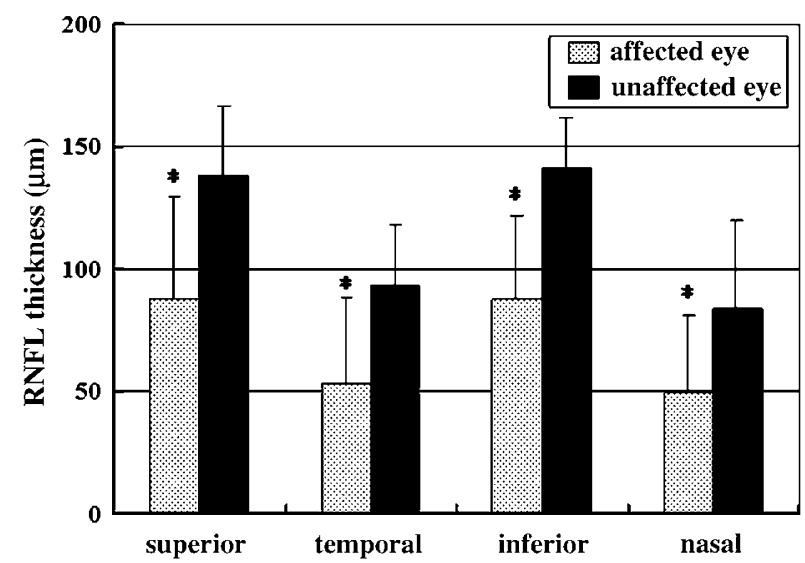

Figure 2 Comparison of peripapillary RNFL thickness in the 22 tested cases. Significant decrease in RNFL thickness is observed in the affected eyes compared with the fellow eyes $(* P<0.001$, Wilcoxon's signed-rank test).

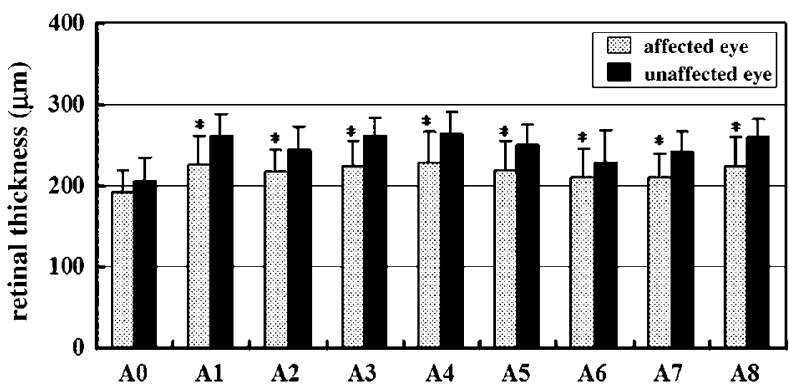

Figure 3 Comparison of macular thickness in the 22 tested cases. Decrease in retinal thickness of the affected eye is prominent in all but A0 areas $\left({ }^{*} P<0.001\right.$, Wilcoxon's signedrank test).

Table 3 Mean values (SD) of the affected to unaffected ratios of macular thickness in each area in eyes with unilateral optic atrophy $(n=22)$

\begin{tabular}{lcc}
\hline Area & Ratio & Range \\
\hline A0 & $0.95(0.12)$ & $0.65-1.17$ \\
A1 & $0.87(0.11)$ & $0.70-1.08$ \\
A2 & $0.89(0.09)$ & $0.73-1.09$ \\
A3 & $0.86(0.09)$ & $0.67-1.01$ \\
A4 & $0.87(0.14)$ & $0.62-1.26$ \\
A5 & $0.87(0.10)$ & $0.68-1.08$ \\
A6 & $0.93(0.25)$ & $0.64-1.89$ \\
A7 & $0.87(0.08)$ & $0.78-1.15$ \\
A8 & $0.86(0.09)$ & $0.66-0.98$ \\
\hline
\end{tabular}

coefficient $=0.094, P=0.668$; Figure 4 ). Concerning the relationship between retinal thickness and the remaining visual field, there was a tendency that eyes with severe visual field loss have the higher degree of retinal thinning (Figure 5). Although the tendency did not reach statistical significance among the four visual field groups

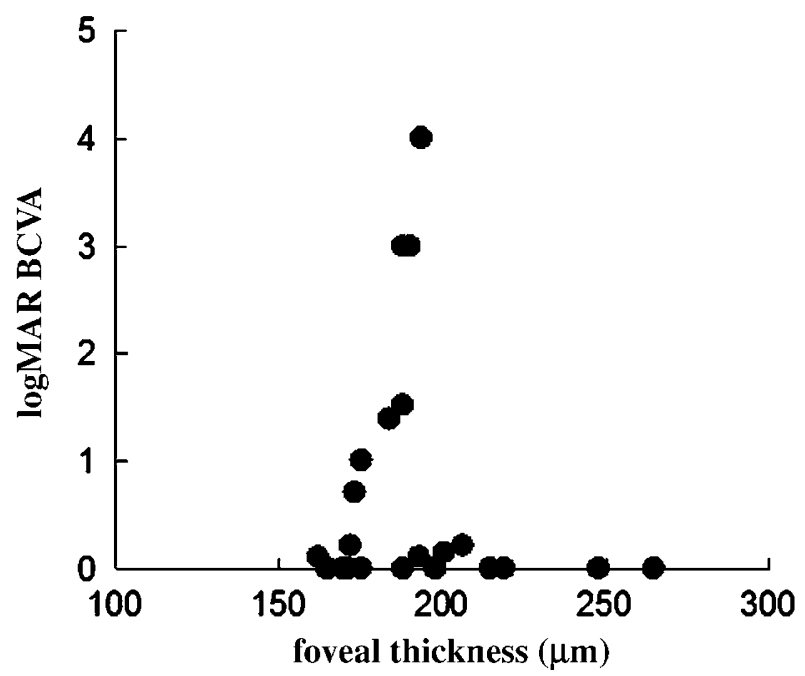

Figure 4 Relationship between foveal thickness (retinal thickness at $\mathrm{A} 0$ ) and $\log$ MAR BCVA in the 22 eyes with optic atrophy. No significant correlation was demonstrated (correlation coefficient $=0.094, P=0.668$, Spearman's analysis).

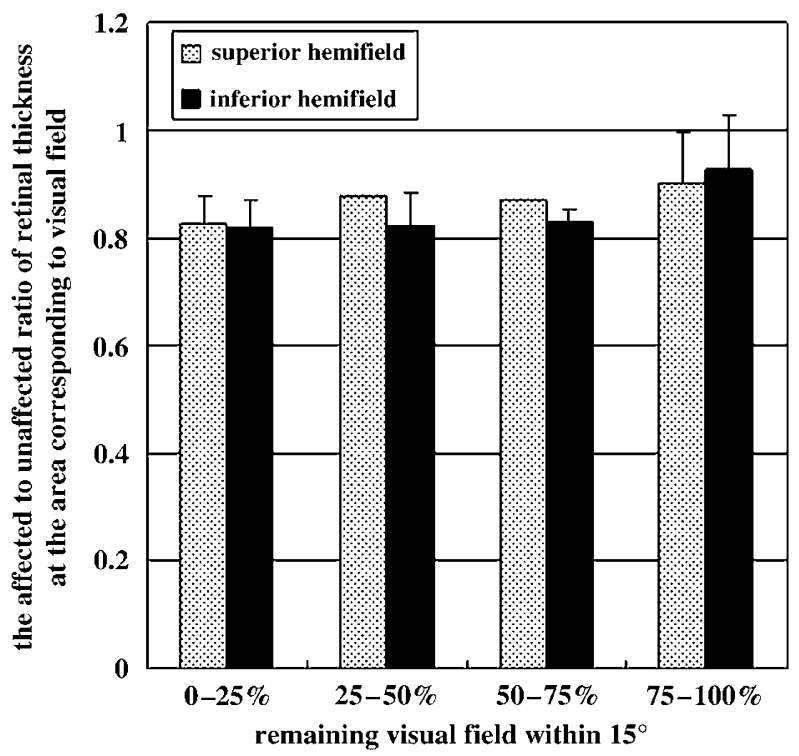

Figure 5 Relationship between the severity of visual field loss and retinal thickness at the corresponding area. The ratio of residual visual field within $15^{\circ}$ was calculated by the area within I-4-e isopter in the superior and inferior hemifields. Note that there is a tendency that eyes with larger remaining visual field have greater retinal thickness.

\section{( $P=0.281$, superior hemifield; $P=0.053$, inferior}

hemifield, respectively), a notable relationship between retinal thickness and the residual visual field was observed when the eyes with equal or more than $50 \%$ remaining visual field was compared with those less than $50 \%$ in the inferior hemifield $(P=0.017$, Mann-Whitney test). 


\section{Discussion}

Previous studies demonstrated scanning laser polarimeter and OCT detected the progressive RNFL thickness reduction in eyes with traumatic optic neuropathy and that in glaucoma, respectively. ${ }^{9-13,19,20}$ Recently, we and Monteiro et al also reported that OCT could detect RNFL thickness reduction corresponding to band atrophy in eyes with compressive lesions at the chiasmal region. ${ }^{14,21}$ In this study, peripapillary RNFL thickness of optic atrophy showed global reduction in all quadrantic sections consistent with previous reports on compressive optic neuropathy and traumatic optic neuropathy. ${ }^{14,19-21}$ Taken together, biometrical assessment of RNFL thickness with OCT reflects the loss of retinal ganglion cell axons in most cases of optic neuropathy.

The measurement of macular thickness in eyes with optic atrophy demonstrated overall decrease with the foveal thickness being unaffected. Given that RNFL is composed of neuroglia, astrocytes, and axons of ganglion cells, and fovea is devoid of ganglion cells, ${ }^{23}$ it is reasonable that macular thickness at $\mathrm{A} 0$, the fovea, did not show significant reduction in eyes with optic atrophy. The ganglion cells and RNFL are reported to contribute to approximately $35 \%$ of the macular thickness. ${ }^{2}$ Accordingly, if ganglion cell loss is the major cause for the macular thinning, macular thickness remains at least about $65 \%$ of controls. This notion was supported by the present finding that the mean macular thickness in eyes with optic atrophy exceeded $60 \%$ of that in unaffected eyes at each area. It has been known that some artefacts in measurement using OCT might have effects on calculated retinal thickness. ${ }^{24}$ However, even taken that into consideration, it would be pertinent from the current data that the degree of retinal thinning is limited. It is noteworthy that even in optic atrophy, the end point status of optic nerve disease, more than $60 \%$ of retinal thickness is preserved, which could not be seen in eyes with severe macular disease. In this regard, simultaneous measurements of macular and peripapillary RNFL thickness are of clinical significance, since these may distinguish retinal disease without fundus abnormality, in which foveal thickness is reported to be reduced, ${ }^{15,25}$ from optic nerve disease and would be useful for the evaluation of macular disease combined with optic neuropathy.

It is known that foveal thickness in some macular diseases demonstrates fine correlation with visual acuity. ${ }^{16-20}$ From this point, it would be an important feature of optic nerve disease that foveal thickness in eyes with optic atrophy does not correlate with BCVA in our study, and be helpful in differential diagnosis. In addition, there was a tendency that an area with severe visual field loss has more marked retinal thinning at the corresponding retinal area. Therefore, measurement of retinal thickness would be useful for the objective assessment of the severity of optic nerve disease.

It is necessary to keep in mind that one of the limitations of our study is the lack of longitudinal evaluation. Additional, large-scale prospective studies are necessary to find out the relationship between the progression of optic atrophy and macular thickness reduction. Another limitation is the small number of subjects. Essentially because of this, we performed the intraindividual comparisons regarding the macular and RNFL thinning in unilateral optic nerve diseases. In patients with systemic diseases such as diabetes and hypertension, macular thickness may be affected by these systemic conditions irrelevant to the primary ocular pathologies. This inevitably increases the SD of macular thickness measured. Thus, interindividual comparisons in a small number of patients were presumed to be difficult to assess the macular thinning in patients with bilateral optic atrophy as compared with independent control subjects.

In conclusion, in eyes with optic atrophy, both macular thickness and peripapillary RNFL thickness were reduced. Macular thickness in the affected eye remained more than $60 \%$ of that in the unaffected eye, and the decrease was not significant in the fovea. Significant correlation was not recognized between foveal thickness and visual acuity. There was a trend that the area of visual field loss corresponded to the retinal thinning area. The characteristic change of macular thickness in optic atrophy would be helpful for the differential diagnosis of retinal and optic nerve diseases.

\section{References}

1 Sadun AA. Optic atrophy and papilledema. In: Albert DM, Jakobeic FA (eds) Principle and Practice of Ophthalmology. (2nd edn.) WB Saunders: Philadelphia, 2000, pp 4108-4116.

2 Zeimer R, Asrani S, Zou S, Quigley H, Jampel H. Quantitative detection of glaucomatous damage at the posterior pole by retinal thickness mapping. A pilot study. Ophthalmology 1998; 105: 224-231.

3 Huang D, Swanson EA, Lin CP, Schuman JS, Stinson WG, Chang W et al. Optical coherence tomography. Science 1991; 254: 1178-1181.

4 Izatt JA, Hee MR, Swanson EA, Lin CP, Huang D, Schuman JS et al. Micrometer-scale resolution imaging of the anterior eye in vivo with optical coherence tomography. Arch Ophthalmol 1994; 112: 1584-1589.

5 Apostolopoulos MN, Koutsandrea CN, Moschos MN, Alonistiotis DA, Papaspyrou AE, Mallias JA et al. Evaluation of successful macular hole surgery by optical coherence tomography and multifocal electroretinography. Am J Ophthalmol 2002; 134: 667-674.

6 Browning DJ, McOwen MD, Bowen Jr RM, O'Marah TL. Comparison of the clinical diagnosis of diabetic macular 
edema with diagnosis by optical coherence tomography. Ophthalmology 2004; 111: 712-715.

7 Spaide RF, Lee JK, Klancnik Jr JK, Gross NE. Optical coherence tomography of branch retinal vein occlusion. Retina 2003; 23: 343-347.

8 Iida T, Hagimura N, Sato T, Kishi S. Evaluation of central serous chorioretinopathy with optical coherence tomography. Am J Ophthalmol 2000; 129: 16-20.

9 Wollstein G, Schuman JS, Price LL, Aydin A, Beaton SA, Stark PC et al. Optical coherence tomography (OCT) macular and peripapillary retinal nerve fiber layer measurements and automated visual fields. $A m \mathrm{~J}$ Ophthalmol 2004; 138: 218-225.

10 Schuman JS, Hee MR, Arya AV, Pedut-Kloizman T, Puliafito CA, Fujimoto JG et al. Optical coherence tomography: a new tool for glaucoma diagnosis. Curr Opin Ophthalmol 1995; 6: 89-95.

11 Schuman JS, Hee MR, Puliafito CA, Wong C, PedutKloizman T, Lin CP et al. Quantification of nerve fiber layer thickness in normal and glaucomatous eyes using optical coherence tomography. Arch Ophthalmol 1995; 113: 586-596.

12 Mistlberger A, Liebmann JM, Greenfield DS, Pons ME, Hoh $\mathrm{ST}$, Ishikawa $\mathrm{H}$ et al. Heidelberg retina tomography and optical coherence tomography in normal, ocular hypertensive, and glaucomatous eyes. Ophthalmology 1999; 106: 2027-2032.

13 Kanamori A, Nakamura M, Escano MF, Seya R, Maeda H, Negi A. Evaluation of the glaucomatous damage on retinal nerve fiber layer thickness measured by optical coherence tomography. Am J Ophthalmol 2003; 135: 513-520.

14 Kanamori A, Nakamura M, Matsui N, Nagai A, Nakanishi Y, Kusuhara S et al. Optical coherence tomography detects characteristic retinal nerve fiber layer thickness corresponding to band atrophy of the optic discs. Ophthalmology 2004; 111: 2278-2283.

15 Kondo M, Ito Y, Ueno S, Piao CH, Terasaki H, Miyake Y. Foveal thickness in occult macular dystrophy. Am J Ophthalmol 2003; 135: 725-728.
16 Degenring RF, Aschmoneit I, Kamppeter B, Budde WM, Jonas JB. Optical coherence tomography and confocal scanning laser tomography for assessment of macular edema. Am J Ophthalmol 2004; 138: 354-361.

17 Goebel W, Kretzchmar-Gross T. Retinal thickness in diabetic retinopathy. A study using optical coherence tomography (OCT). Retina 2002; 22: 759-767.

18 Sanchez-Tocino H, Alvarez-Vidal A, Maldonado MJ, Moreno-Montanes J, Garcia-Layana A. Retinal thickness study with optical coherence tomography in patients with diabetes. Invest Ophthalmol Vis Sci 2002; 43: 1588-1594.

19 Medeiros FA, Susanna Jr R. Retinal nerve fiber layer loss after traumatic optic neuropathy detected by scanning laser polarimetry. Arch Ophthalmol 2001; 119: 920-921.

20 Medeiros FA, Moura FC, Vessani RM, Susanna Jr R. Axonal loss after traumatic optic neuropathy documented by optical coherence tomography. Am J Ophthalmol 2003; 135: 406-408.

21 Monteiro ML, Leal BC, Rosa AA, Bronstein MD. Optical coherence tomography analysis of axonal loss in band atrophy of the optic nerve. Br J Ophthalmol 2004; 88: 896-899.

22 Kusuhara S, Teraoka Escano MF, Fujii S, Nakanishi Y, Tamura Y, Nagai A et al. Prediction of postoperative visual outcome based on hole configuration by optical coherence tomography in eyes with idiopathic macular holes. Am J Ophthalmol 2004; 138: 709-716.

23 Sjostrand J, Popovic Z, Conradi N, Marshall J Morphometric study of the displacement of retinal ganglion cells subserving cones within the human fovea. Graefes Arch Clin Exp Ophthalmol 1999; 237: 1014-1023.

24 Jaffe GJ, Caprioli J. Optical coherence tomography to detect and manage retinal disease and glaucoma. Am J Ophthalmol 2004; 137: 156-169.

25 Jacobson SG, Cideciyan AV, Huang Y, Hanna DB, Freund $\mathrm{CL}$, Affatigato LM et al. Retinal degenerations with truncation mutations in the cone-rod homeobox (CRX) gene. Invest Ophthalmol Vis Sci 1998; 39: 2417-2426. 